\title{
Diagnostic and Treatment Algorithms of Pancreatic Cystic Tumors
}

\author{
Chair: \\ Jens Wernera \\ $\begin{array}{lll}\text { Participants: Orlin Belyaev } & \text { Carlos Fernández-del Castillo }^{c} & \text { Philippe Lévy }^{d} \quad \text { Matthias Löhr }^{\mathrm{e}} \\ \text { Julia Mayerle }^{f} & \text { Waldemar Uhl }^{\mathrm{b}}\end{array}$ \\ a Department of General, Visceral, and Transplantation Surgery, Ludwig Maximilians-University, Munich, Germany; \\ ${ }^{b}$ Department of General and Visceral Surgery, St. Josef-Hospital, Ruhr-University Bochum, Bochum, Germany; \\ 'Department of Surgery, Massachusetts General Hospital and Harvard Medical School, Boston, MA, USA; \\ ${ }^{\mathrm{d}}$ Department of Gastroenterology and Pancreatology, Pôle des Maladies de I'Appareil Digestif, DHU Unity, Hôpital Beaujon, Faculté Denis \\ Diderot, AP-HP, Clichy, France; \\ e Department of Clinical Science, Intervention and Technology (CLINTEC), C1:77, Gastrocentrum, Karolinska Institutet, Stockholm, Sweden; \\ ${ }^{f}$ Department of Gastroenterology and Hepatology, Ludwig Maximilians-University, Munich, Germany
}

\section{Question 1: What kind of initial diagnostics do you recommend after a pancreatic cyst is detected by abdominal ultrasound?}

Belyaev/Uhl: The initial diagnostic evaluation of a newly detected pancreatic cystic lesion (PCL) should identify the specific type of PCL and its individual risk of malignancy as accurately as possible. It includes a comprehensive structural and functional assessment of the pancreas. Besides family history for malignancy, standard physical examination, and complete laboratory workup including carbohydrate antigen (CA) 19-9 and carcinoembryonic antigen (CEA), fecal elastase, and OGTT, dedicated pancreas protocol imaging by means of computed tomography (CT) and magnetic resonance imaging (MRI)/magnetic resonance cholangiopancreatography (MRCP) play a central role. MRI is superior in detecting size, number, and pancreatic duct connection of PCLs and avoids radiation exposure, while CT is more reliable in detecting calcifications, solid nodules, and concomitant pancreatic cancer.

Fernández-del Castillo: I perform an MRI of the pancreas with MRCP.

Lévy: Three phases CT scan without and with iodine IV contrast (arterial and portal sequences), water digestive contrast, MRCP (thick T2 slices centered on the main pancreatic duct (MPD) mandatory). Endoscopic ultrasound (EUS) is optional and rare. Fine needle aspiration (FNA) is exceptional.

Löhr: An MRI with MRCP and, at the first instance, with secretin stimulation (this is important to assess any connection with the duct system but not necessary on subsequent controls).
Mayerle: Besides taking a detailed history to exclude high-risk stigmata and worrisome features, to me there are two questions to be answered for risk assessment of a cystic pancreatic lesion, namely a connection to the pancreatic duct present only in intraductal papillary mucinous neoplasm (IPMN) and pancreatic pseudocysts, best answered by MRI and MRCP, as well as solid components and CEA elevation in the cystic fluid, best answered by EUS and FNA if the cyst is larger than $2 \mathrm{~cm}$ in diameter.

\section{Question 2: How can the malignant potential of a pancreatic cyst be predicted?}

Belyaev/Uhl: Almost all PCLs have a certain malignant potential, and there is no single absolute sign of malignancy. The individual risk should be determined by an experienced pancreatologist, and all available data should be taken into account. PCL size of $>4 \mathrm{~cm}$, rapid growth $>5 \mathrm{~mm}$ /year, MPD involvement and dilatation $>5 \mathrm{~mm}$, contrast-enhancing mural nodules or other solid components, jaundice, and increased CA 19-9 should be considered as strong stigmata of cancer transformation. A positive own history of the patient or family history for adenocarcinoma (pancreas, breast, ovaries, gastrointestinal tract), new-onset diabetes mellitus, recurrent pancreatitis, and weight loss may be considered additional risk factors of lower specificity.

Fernández-del Castillo: Any pancreatic cyst has the potential to become malignant over time, and at present we have no way to predict which ones will progress (which are the minority) and which ones will not (the vast majority). We do have parameters that allow

\section{KARGER}

() 2018 S. Karger GmbH, Freiburg

Fax +497614520714
Prof. Dr. med. Jens Werner

Klinik für Allgemein-, Viszeral-, Gefäß- und Transplantationschirurgie

LMU Klinikum der Stadt München

Marchioninistraße 15, 81377 München, Germany

jens.werner@med.lmu.de 
us to determine which cysts have a higher likelihood of harboring malignancy (either high-grade dysplasia or invasive cancer). These parameters are based mostly on morphology of the cysts (size, presence of solid component, associated dilation of the pancreatic duct) but also on symptoms (jaundice, pancreatitis, recent-onset diabetes).

Lévy: Serous cystic neoplasm (SCN): never malignant; mucinous cystic neoplasm $(\mathrm{MCN})$ : size $>4 \mathrm{~cm}$ and mural nodules; IPMN: MPD diameter larger than $9 \mathrm{~mm}$, mural nodules $>5 \mathrm{~mm}$, solid mass. CA 19-9 is not useful. Diffusion restriction on MRI; solid pseudopapillary neoplasm: all (locally) malignant.

Löhr: In case of main-duct IPMN: virtually all will eventually convert to malign. In case of side-branch IPMN: size (>30 mm) and worrisome features (solid contrast-enhancing mural nodule $>$ $5 \mathrm{~mm}$; MPD > $5 \mathrm{~mm}$; CA 19-9 > $37 \mathrm{U} / \mathrm{ml}$ ) in line with the recent guidelines (Gut 2018).

Mayerle: One has to be aware that the diagnostic accuracy of cystic pancreatic lesions when all possible imaging modalities are used is only $80 \%$, and thus age, comorbidity, and risk of surgery have to be taken into account if a clinical decision is taken. The best predictor of malignancy is not morphology but clinical symptoms such as new-onset diabetes, weight loss, bouts of pancreatitis, or jaundice. With respect to morphology, solid nodules are still the best predictor, and size or growth is second best.

\section{Question 3: What is the indication for puncturing the cyst and to perform an analysis of the cyst fluid? Which parameters are you interested in?}

Belyaev/Uhl: EUS-FNA has a very low diagnostic yield of $8-59 \%$, may be technically demanding, and its specific complications, such as acute pancreatitis, bleeding, and tumor seeding, despite being rare, may have a profoundly negative effect on the management strategy. In most cases, the latter is determined by the combination of clinical symptoms and imaging findings. Thus, we try to avoid EUS-FNA whenever possible. This diagnostic procedure also requires a very experienced gastroenterologist who is not available everywhere. Therefore, it should only be used in cases where its findings would change the management. Measurements of cyst fluid CEA, CA 19-9, amylase, and lipase should be combined with cytology with KRAS/GNAS mutation analysis.

Fernández-del Castillo: I recommend EUS with FNA of the cyst fluid as well as biopsy of the cyst wall in cysts that are greater than 2 $\mathrm{cm}$ in size or in patients with larger cysts in whom we would normally recommend surgery but that for reasons of age or comorbidities should not have the operation unless we have a higher suspicion or certainty of malignancy. EUS gives more details on the morphology of the cyst, and the cytology of the fluid or the assessment of the biopsy of the cyst wall can assist in making a more precise diagnosis of the type of cyst and also on the presence of malignancy. We also send the fluid for genetic analysis. Currently, this is useful to make a more precise diagnosis, but not to confirm or exclude malignancy (although much progress is being made in this regard). One can also measure CEA in the cyst fluid. This is present in about $70 \%$ of IPMNs and MCNs, but always low in serous cystadenomas and almost always low in cystic neuroendocrine tumors and inflammatory cysts.

Lévy: This is less and less useful considering the progress of imaging, especially MRCP. It is mainly used in case of difficult diagnosis between macrocystic unique cyst compatible with either SCN or MCN. It is not useful at all in IPMN. CEA, lipase, and cytology should be measured (and histology, if possible).

Löhr: Pulling out the needle and seeing mucus is one feature, then immunocytochemistry for mucins ((MUC 1 vs. 5A); CEA > $182 \mathrm{U} / \mathrm{ml})$

Mayerle: In a recent meta-analysis, cytological analysis of cyst fluid had $42 \%$ sensitivity and $99 \%$ specificity for differentiating mucinous from non-mucinous pancreatic cystic neoplasm (PCN). A cyst fluid CEA level of $\geq 192 \mathrm{ng} / \mathrm{ml}$ can distinguish mucinous from non-mucinous cysts, with a sensitivity of $52-78 \%$ and specificity of $63-91 \%$. Therefore, the negative predictive value to exclude a mucinous lesion is very high, and thus, in my view, this helps in decision making. In general, combination tests, such as EUS morphology, cytology, and cyst fluid CEA, provide greater accuracy in detecting mucinous PCN than either EUS morphology or cytology alone.

\section{Question 4: Which patient does not need any further assessment and which does not need any follow-up?}

Belyaev/Uhl: Follow-up makes no sense and may be discontinued in patients who determinedly reject surgery and in those who are not fit for surgery because of comorbidities. All other patients need lifelong follow-up irrespective of the type of management.

Fernández-del Castillo: Patients in whom we have a definitive diagnosis of a serous cystadenoma (because of typical morphology or based on the results of the EUS-guided biopsy), which is almost universally benign. Also, in patients in whom an operation would not be considered under any circumstance because of patient preference or comorbidities.

Lévy: SCN: follow-up at 1 year and then symptom-based followup; MCN: follow-up if diameter $<4 \mathrm{~cm}$ and no mural nodules (to be validated by prospective long-term studies); IPMN: follow-up mandatory in all.

Löhr: Solitary benign cysts without connection to the duct system; all patients unfit for surgery; small side-branch IPMN $<5 \mathrm{~mm}$ without tendency to grow over 10 years. 
Mayerle: I entirely agree with the criteria mentioned above and would only add that if comorbidity or age preclude surgical therapy, then follow-up is symptom-based.

\section{Question 5: How often do patients with branch-duct IPMNs need a re-evaluation, and what kind of investigation do you perform?}

Belyaev/Uhl: Timing for re-evaluation of branch-duct IPMNs without high-risk stigmata and worrisome features at the time of the initial presentation depends on their size. Cysts $<2 \mathrm{~cm}$ should be reassessed after 6 months and when findings are stable, intervals may lengthen to 1 and 2 years over time. Branch-duct IPMNs $>2$ $\mathrm{cm}$ should be initially reassessed after 3 months, and if no changes appear the intervals may lengthen to 6 or 12 months. If a high-quality CT was initially performed, further imaging controls may be based on MRI/MRCP and tumor markers only until changes occur.

Fernández-del Castillo: We repeat cross-linear imaging (typically MRI, unless this cannot be done for some reason) 6 months after the initial diagnosis, and then yearly, unless the cyst is less than $1 \mathrm{~cm}$, in which case we do it every 2 years.

Lévy: At 1 year after diagnosis. The further follow-up rhythm depends on the size. Smaller than $2 \mathrm{~cm}$ : every 2 years; $>2 \mathrm{~cm}$ : every year. Alternating MRI and CT scan. EUS if modification of symptoms or images.

\section{Löhr: Yearly MRI.}

Mayerle: To sufficiently answer this question, I would like to turn the readers' attention to the recently published European guidelines on PCN which suggest evidence-based follow-up algorithms (Gut 2018;67:789-804). In addition, the revised Fukuoka consensus guidelines published in 2017 give valid recommendations for follow-up.

\section{Question 6: Are there any biomarkers which you rely on during follow-up of unresected patients with IPMN?}

Belyaev/Uhl: No reliable biomarkers of malignant transformation of IPMN exist up to date. However, measurements of CA 19-9 and CEA are non-invasive as well as inexpensive, and may be indicative of malignant transformation, so they belong to our routine surveillance workup of unresected IPMN. However, the future lies in socalled 'liquid biopsies' with the determination of circulating markers (cfDNA) for malign transformation of IPMN and MCN in the blood.

Fernández-del Castillo: I obtain serum levels of CA 19-9 and CEA, as well as hemoglobin A1C at baseline, and then repeat them if I see changes in the cyst during follow-up.
Lévy: Not yet, and certainly not CA 19-9 which is the poorest marker ever in terms of sensitivity and specificity. CA 19-9 should be discarded.

Löhr: Currently, no biomarkers are available. Serum CA 19-9 $(<37 \mathrm{U} / \mathrm{ml})$ may be indicative.

Mayerle: In my view, symptoms are in many instances not appreciated sufficiently, and it is always a combination of symptoms, imaging, and laboratory workup leading to a clinical decision for follow-up.

\section{Question 7: Do you vary your treatment} recommendation according to the localization (head/neck, tail) of the cyst?

Belyaev/Uhl: Localization of PCLs has not been shown to correlate with the presence of high-risk stigmata or worrisome features, so in principle it has no effect on the treatment recommendation alone. However, cysts located in the head of the pancreas tend to be symptomatic more often (pancreatitis, cholestasis, jaundice, ...), so the chance of recommending surgery in those cases is higher.

Fernández-del Castillo: I do not vary my recommendations based on the location of the cyst, since the decision to proceed with resection is based on the risk of malignancy and not on the risk of the operation.

Lévy: In a minor way, yes. Decision on left pancreatectomy is 'easier' than Whipple procedure. As far as possible, limited resection should be discussed (enucleation, ablation of uncus, central pancreatectomy).

Löhr: As a non-surgical expert, I can only relate the experiences from my surgical colleagues: of course. Multifocal IPMNs may be in need of a total pancreatectomy. Others may be done as an enucleation, laparoscopic or robotic.

Mayerle: I agree.

\section{Question 8: Are local resections helpful in some cases? Please define when you would recommend a limited organ-sparing resection (e.g. enucleation, segmental resection, etc.).}

Belyaev/Uhl: Symptomatic lesions and those with suspected malignancy should undergo standard oncologic resection depending on their localization - pancreatoduodenectomy, distal pancreatectomy, or total pancreatectomy. IPMNs with high-risk stigmata and worrisome features originating from the pancreatic duct system have a favorable prognosis only after oncologic resections, so these PCLs are no candidates for parenchyma-sparing procedures (PSP) 
such as enucleation. PSP may be offered to highly selected patients who are young and fit enough to survive this complicated surgery in order to decrease their long-term risk of diabetes and exocrine insufficiency and thus increase their quality of life. In this respect, some ideal patients with Frantz's tumor in the central part of the pancreas are suitable for oncologic segmental resection. Additionally, PSP are oncologically reasonable in small pancreatic neuroendocrine tumors, SCNs, and MCNs $<2 \mathrm{~cm}$ (only if there is suspicion of malignancy) and technically feasible for small lesions, which are superficially localized in easily accessible parts of the pancreas, do not communicate with the MPD, and are distant enough to it to allow safe resection without iatrogenic duct lesions.

Fernández-del Castillo: I do not think that an enucleation is a proper operation for an IPMN. Once again, if the decision to remove it is because the risk of malignancy is higher, then a proper oncologic resection should be performed. By definition, an enucleation of a branch-duct IPMN is leaving behind part of the branch duct that is connecting to the main ductal system. I do middle or central pancreatectomies for branch-duct IPMNs, and carefully check the margins intraoperatively.

Löhr: As stated above - yes! Certainly not in main-duct IPMN as in this case one has to consider an oncological procedure in any event.

Mayerle/Lévy: As far as possible yes, depending on the suspected pathological results (no limited resection in case of suspicion of malignancy) and the location, especially the anatomical relationship with MPC (need of an excellent MRCP to be discussed).

\section{Question 9: What development is most needed for safe treatment of patients with cystic tumors of the pancreas?}

Belyaev/Uhl: Safe treatment of PCLs has two different aspects. On the one hand, discovery of reliable early biomarkers of malignant transformation may be crucial for determining the treatment strategy. Development of liquid biopsy for detection of malignant PCLs may help to precise indications for surgery in the future. On the other hand, PCLs are associated with soft pancreatic tissue, and thus their resection comes along with very high rates of postoperative pancreatic fistula (POPF). Thus, innovations in surgical technique aimed at reducing POPF may considerably increase the safety of surgical treatment of PCLs.

Fernández-del Castillo: Because so many patients are now found to have pancreatic cysts, doing surveillance with MRI or CT is very expensive and cumbersome. A blood-based test that could triage patients with no risk would be a major advance, since they could get this periodically, reserving the imaging for patients who do have a risk. This blood test could be based on circulating tumor cells or exosomes.

Lévy: Blood biomarkers to avoid repeated imaging procedures!

Löhr: Biomarkers indicating early malignant transformation; better localization/extension (pre-/intraoperative Spyglass, maybe confocal laser endomicroscopy); further development of minimally invasive/minimally traumatic surgical techniques.

Mayerle: We need to work on increasing the diagnostic accuracy of cystic pancreatic lesions to improve the patients' care. We need to publicly report that the risk of malignancy in PCLs is lower than previously reported and to give the all-clear to fearful patients whenever possible.

\section{Participants}

PD Dr. med. Orlin Belyaev

Department of General and Visceral Surgery

St. Josef-Hospital, Ruhr-University Bochum Gudrunstraße 56, 44791 Bochum, Germany o.belyaev@klinikum-bochum.de

Carlos Fernández-del Castillo, MD

Department of Surgery

Massachusetts General Hospital and Harvard Medical School

15 Parkman Street, Boston, MA 02114-3117, USA

cfernandez@partners.org

Prof. Philippe Lévy

Service de Pancréatologie-Gastroentérologie

Pôle des Maladies de l'Appareil Digestif, DHU Unity

Hôpital Beaujon, AP-HP

92118 Clichy Cedex, France

philippe.levy@aphp.fr

Matthias Löhr, MD, MD PhD, FEBG, AGAF

Department of Clinical Science, Intervention and Technology (CLINTEC)

C1:77, Gastrocentrum, Karolinska Institutet

14186 Stockholm, Sweden

matthias.lohr@ki.se

Prof. Dr. med. Julia Mayerle

Medizinische Klinik und Poliklinik 2

Klinikum der LMU München

Marchioninistraße 15, 81773 München, Germany

julia.mayerle@med.uni-muenchen.de

Prof. Dr. med. Waldemar Uhl

Department of General and Visceral Surgery

St. Josef-Hospital, Ruhr-University Bochum

Gudrunstraße 56, 44791 Bochum, Germany

w.uhl@klinikum-bochum.de 